\title{
Multi-Criteria Assessment Problems in Breast Cancer Surgery
}

\author{
Cornel Resteanu ${ }^{1}$, Romica Trandafir ${ }^{2}$, Xenia Bacinski ${ }^{3}$ \\ ${ }^{1}$ National Institute for Research and Development in Informatics \\ 8-10, Averescu Avenue, 011455, Bucharest 1, ROMANIA, \\ resteanu@ici.ro \\ 2 Technical University of Civil Engineering \\ 24, Lacul Tei Avenue, 020396, Bucharest 2, ROMANIA, \\ romica@utcb.ro \\ ${ }^{3}$ Institute of Oncology \\ 52, Fundeni Avenue, 022338, Bucharest 2, ROMANIA, \\ xenia_bacinschi@yahoo.com
}

\begin{abstract}
It is well known that in the breast cancer surgery, of paramount importance is to choose the best surgery type according to the patient's disease stage. The best choice is possible to be made by computing, in the Multiple Attribute Decision Making paradigm, the merits of all types of surgery that can be applied. The main issues are the diverse assessment problems with conflicting criteria and different measure units. Therefore both the physician and the patient have a good scientific tool in their practical decision process.
\end{abstract}

Keywords: Breast cancer, Surgery schema, Merits’ Assessment, Multiple Attribute Decision Making, Decision Analysis.

\section{Introduction}

By definition, "Neoplasm or tumor represents the abnormal cells excessive proliferation that resembles more or less the tissue in which they are growing and they end up obtaining a biological autonomy". Neoplasm has miscellaneous causes: hereditary, chemical (tabacosis), physique (sun radiations), biologic (a virus action). These causes can join together. Sometimes, a tumor can have an unknown cause. Tumor cells lost the sensibility to organic structure that normally discourages any excessive proliferation. Immune system's white cells inhibit or destroy all isolate tumor cells that appear in the case of a healthy individual. Therefore, a real tumor could grow only if its cells became resistant to immune system.

There are two kinds of tumor, benign tumor and malignant (cancerous) tumor:

- Benign tumors usually have a limited volume. They repress to neighbor tissues without invading them, they do not produce metastases and, in most of the cases, their consequences are not serious;

- Malignant tumors, i.e. cancerous tumors, have opposite characteristics to benign tumors. They often become bulky and ill bounded. They infiltrate the surrounding tissues, relapse after the ablation and, especially, they have the tendency to expand and form metastases.

Breast neoplasm [1] is the most frequently woman cancer type, representing about $29 \%$ of new annual diagnosed cancer. It represents the first cause of death among women younger than age 55. The breast neoplasm incidence has significantly increased in the last decades, but mortality decreased because the disease early detection and modern treatments.

The disease stages are the following:

- Stage I is known as precocious stage, early stage;

- Stages IIA and IIB are considered advanced loco regional stages; the tumor is smaller than 5 centimeters and can not include the breast adjacent tissues;

- Stages IIIA and IIIB are considered advanced loco regional stages; the tumor is bigger than 5 centimeters and can include the breast adjacent tissues;

- Stage IV, metastasis, the cancer cells go and proliferate in different organs situated at a distance from the primary tumor. 
The treatment depends on disease stage. It is limited to surgery associated with radiotherapy and hormonal-therapy in early stages and can become more complex and aggressive in late stages by using, beside surgery, cytostatics, monoclonal antibodies, radio-therapy, hormonal-therapy, an entire arsenal of symptomatic therapies in order to ensure a better life quality.

In this paper, the focus is on surgical treatments' assessment, computing merits of the surgery schema. It is possible to use techniques like in [2] but they have some limitations. In this paper, merits are computed using the Multi-Attribute Decision Making (MADM) paradigm. In the following, there are some theoretical considerations on mathematical instruments.

For objects in a given set, the MADM methods [3, 4] compute, in the assessment process, its merits, values between 0 and 1, used for objects' ranking or / and optimal choice. The objects' set are finite and explicit done. The assessment is made upon a set of objects' attributes.

The second section of the paper presents the breast cancer surgery model and its instance in conformity with the experiment made. Some general methods to solve the assessment generated problems, based on the previously mentioned model, are given in the third section. These methods are presented in the natural language, but one of them is presented in mathematical language. Also, the general procedure, for solving multiattributes-states_of_nature-experts problems, is done in the same section. Model's consistency analysis and assessment numerical results with comments on their significance are given in the fourth section. Some conclusions, given in the fifth section, end this paper.

\section{Breast Cancer Surgery Model}

This paper approaches the "Breast cancer surgery model" through the agency of a tool named OPTCHOICE [5, 6]. OPTCHOICE software may be characterized as a pervasive optimization service. Recall that an Internet service is pervasive if it is available to any client, free of charge, anywhere, anytime and without delay.

Web enabled optimization is a new trend in

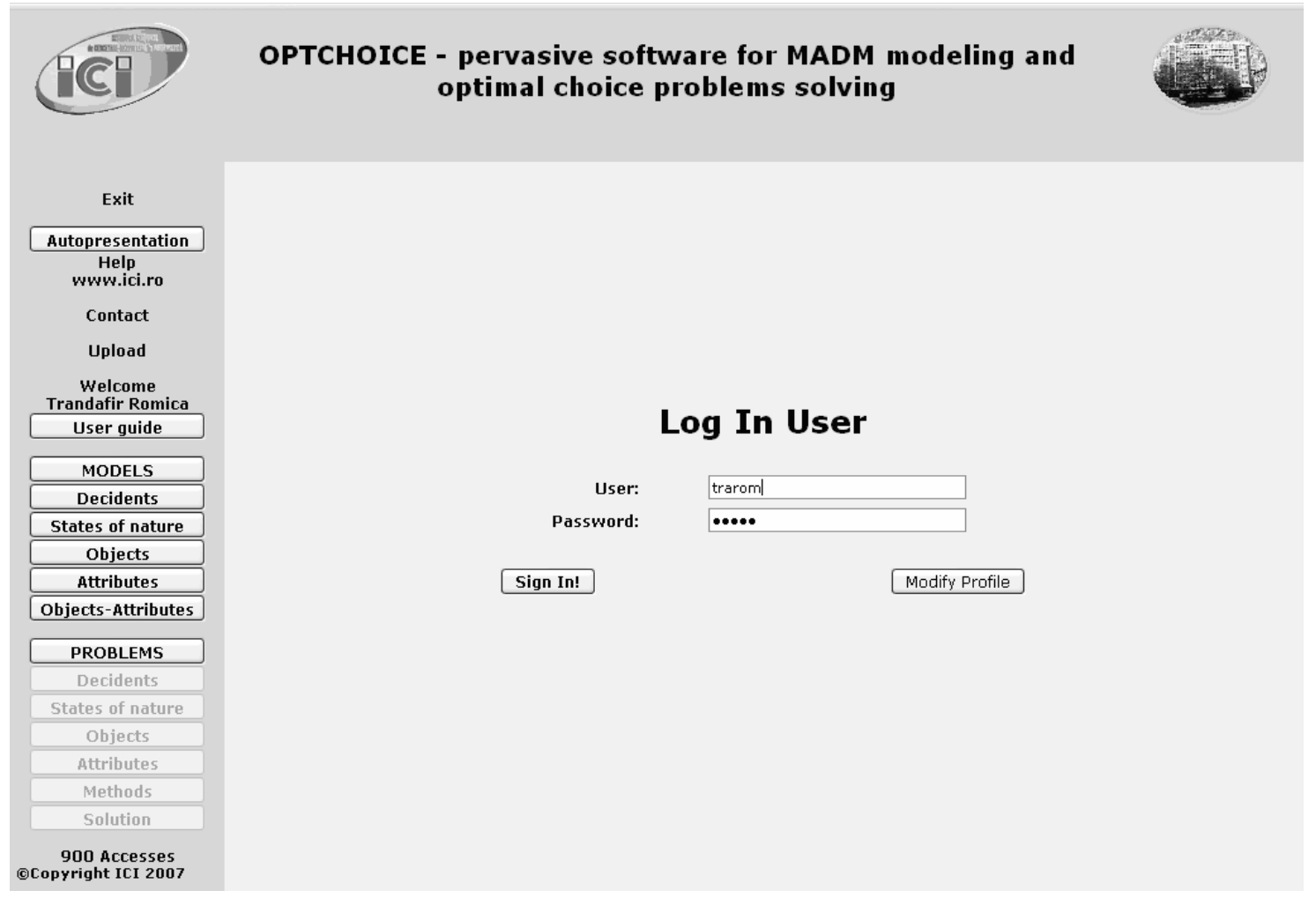

Capture 1. The front page of OPTCHOICE software 
treating Operations Research problems over the Internet. Part of this new trend, the OPTCHOICE software is one of the first Internet-based programs designed to describe MADM mathematical models, define Assessment and Decision Problems (ADPs) on them, and solve these problems in informatics performance conditions.

The above named model, as general and OPTCHOICE-instanced model, involves the following elements:

- $\quad D=\{d(k) \mid k=\overline{1, \boldsymbol{k}}\},(\boldsymbol{k}=\operatorname{card}(D))$, a set of experts (decision-makers), whose elements are the persons with assignments in defining the model and $W D=\{w d(k) \mid k=\overline{1, \boldsymbol{k}}\}, \boldsymbol{k}=\operatorname{card}(D)$ the set of corresponding weights.

The application considers as experts (decision-makers) three physicians, specialists in breast cancer, and thus $D=\{d(k) \mid k \in \overline{1,3}\}=$ \{medical and radiology oncologist, medical oncologist, surgeon oncologist $\}$, with the following weights in the assessment process:

$W D=\{w d(k) \mid k=\overline{1,3}\}=\{40 \%, 30 \%, 30 \%\}$.

- $\quad S=\{s(l) \mid l=\overline{1, l}\},(l=\operatorname{card}(S))$, a set of states of nature, each one of them synthetically signifying the totality of circumstances that determines variations in the model, and $W S=\{w s(l) \mid l=\overline{1, l}\}, \boldsymbol{l}=\operatorname{card}(S)$ the set of corresponding weights.

The application considers as states of nature six disease stages $S=\{s(l) \mid l=\overline{1,6}\}=\{$ stage I, stage IIA, stage IIB, stage IIIA, stage IIIB, stage $I V\}$, with the following weights in the assessment process: $W S=\{w s(l) \mid l=\overline{1,6}\}=$ $\{10 \%, 15 \%, 15 \%, 25 \%, 25 \%, 10 \%\}$.

- $O=\{o(i) \mid i=\overline{1, \boldsymbol{i}}\}, \quad(\boldsymbol{i}=\operatorname{card}(O))$, a set of objects containing the entities subject to assessment process.

The application considers as objects four surgery schema $O=\{o(i) \mid i=\overline{1,4}\}=\{$ breast conserving surgery, removal of the primary tumor-lumpectomy, palliative mastectomy, radical mastectomy?.
- $A=\{a(j) \mid j=\overline{1, j}\},(j=\operatorname{card}(A))$, a set of attributes, consisting in those characteristics which are evaluated for every object and $W A=\{w a(j) \mid j=\overline{1, j}\}$ the set of corresponding weights. The attributes have a senses vector $S A=\{s a(j) \mid j=\overline{1, \boldsymbol{j}}\} \quad$ whose elements are maximum or minimum. Each attribute $a(j), \quad j=\overline{1, j}$ has a variation interval [pes_a(j),opt_a(j)].

The application considers as attributes some important characteristics for surgery schema, $A=\{a(j) \mid j=\overline{1,3}\}=$ \{curative effects, side (uncalled) effects, costs\}. For a given expert (decision-maker) and a state of nature:

1. The elements corresponding to the first attribute are the success rate assessed for all surgeries made in a given past period of time;

2. The elements corresponding to the second attribute are the grades assessed for cases without important side effects calculated over all surgeries made in the same period of time;

3. The elements corresponding to the third attribute are the norm costs $+/-$ the cost due to the particularities in the surgery's performing.

The corresponding weights are: $W A=$ $\{w a(j) \mid j=\overline{1,3}\}=\{60 \%, 15 \%, 25 \%)$. The senses vector is \{maximum, maximum, minimum $\}$. The first two attributes are expressed in grades (the greater the grade the better the corresponding attribute). The third attribute is expressed in Romanian currency (the smaller is the cost, the convenient it is). For the first two attributes the variation interval is $[1,10]$, in grades, given by the experts, and for the third attribute the variation interval is [1000, 40000], in RONs, conform to the medical unit's catalog.

The consequences matrix $C: D \times S \times O \times A$ $\rightarrow \Re$, where the element $C(d(k), s(l), o(i)$, $a(j))=c_{k l i j}$ represents the evaluation of the attribute $a(j)$ for the object $o(i)$, in the opinion of the expert (decision-maker) $d(k)$, in the 
state of nature $s(l)$, with $k=\overline{1, \boldsymbol{k}}, \quad l=\overline{1, l}$, $i=\overline{1, \boldsymbol{i}}, j=\overline{1, \boldsymbol{j}}$.

The application considers as consequences those $c_{k l i j}$ with $k=\overline{1,3}, l=\overline{1,6}, i=\overline{1,4}, j=\overline{1,3}$.

In the following table one can see, for example, a consequences matrix:

Table 1. Data corresponding to the first physician for the first stage

\begin{tabular}{|l|l|l|l|}
\hline$C$ & $a(1)$ & $a(2)$ & $a(3)$ \\
\hline$o(1)$ & 10 & 10 & 2000 \\
\hline$o(2)$ & 10 & 9 & 1800 \\
\hline$o(3)$ & 10 & 2 & 1800 \\
\hline$o(4)$ & 10 & 2 & 1500 \\
\hline
\end{tabular}

In fact, target model has $3 \times 6=18$ consequences matrices. Such kind of model can generate a lot of assessment problems. The above presented model has generated the following classes of problems: 1) every physician for every disease stage $(3 \times 6=18$ problems), 2) all physicians for every disease stage ( $1 \times 6=6$ problems) and 3$)$ all physicians for all disease stages $(1 \times 1=1$ problem $)$.

\section{Assessment Problems' Solving}

A particular problem may be solved by using many methods. There are essentially two classes of solving methods: first, methods that produce explicit object evaluations (by using a set of such methods, one associate to each object an evaluation vector) and second, methods that produce object characteristics (these analysis methods associate to each object a matrix of discriminators). OPTCHOICE implements ten methods from the first class, namely the maximax, maximin, non-dominance, linear utility function, scores, diameters, Onicescu, Pareto, TOPSIS, TODIM methods, in conjunction with several normalization methods, and six methods from the second class, namely methods of dominance analysis: for every object, the number of dominated objects, for every object, the number of objects which dominate $i t$, the minimal/maximal number of characteristics through the objects are the best evaluated, the minimal/maximal number of characteristics through the objects are the worse evaluated.
Since each evaluation method reflects a different point of view about assessment and optimality, it is clear that applying different methods to the same set of data will often lead to different solutions.

A procedure implemented in OPTCHOICE addresses this problem; it proposes also a global solution by processing the results stored in the evaluation vector and possible in the matrices of discriminators.

\section{Chosen Methods for Assessment}

Breast cancer surgery problems were multiple solved, taking into account the following methods: LINEAR UTILITY FUNCTION (M1), TOPSIS (M2), SCORES (M3) and TODIM (M4), data being normalized with the first von Neuman - Morgenstern method. In order to see the complexity of these methods, one presents the methods in natural language.

1. LINEAR UTILITY FUNCTION method. This method, as its name shows, is based on linear expresed utility function. It is interesting to say that the MADM domain starts with so kind of functions' computing. Though very simple, the method leeds to results close to those mentaly assessed by the experts in decision making. One works with normalized consequences matrix and the weigths of attributes. The function's value, for a given object, is the sum, after all atributes, of products between its attributes' values and the weigths of attributes. Obviously, the function's values rank the set of objects and one can point the optimum.

2. TOPSIS method. It computes a composite merit starting from two distances. The first distance is like in Pareto method i.e. the distance between the objects and the positive ideal point that would represent an object characterized by the attributes whose values are optimum, if the sense of the attribute is maximum, or pessimum, if the sense of the attribute is minimum. The second distance is the distance between the objects and the negative ideal point that would represent an object characterized by the attributes whose values are pessimum, if the sense of the 
attribute is maximum, or optimum, if the sense of the attribute is minimum. In the method authors opinion, the distance taken into consideration must be the one resulted from L2 norm, that means the Euclidian distance; this distance gives a better measure in distance computing. Obviously, all objects' merits are computed with a formula in such a way which ensures that their values are situated between 0 and 1 .

3. SCORES method. Scores method uses a complex algorithm based on rankings of the objects vectors depending on their attributes. Objects that contain attributes equal to maximum values in consequences matrix, if their sense is maximum, or equal to minimum values in consequences matrix, if their sense is minimum, receive the greatest scores. Other positions in the ranking bring lower scores, depending on objects occupied rank in a certain ranking. By totalizing the won scores of each object a score is obtain. At the end, normalized scores as merits are built.

4. TODIM method. To achieve the computing of objects merits, this method needs the attributes relative importance, which is not entry data in this case. But the OPTCHOICE system can compute these data starting from the existing data. The method computes objects' total weights for each attribute based on relative importance set and it considers the index that maximum is achieved. Using this index, one computes the absolute dominances between any two objects, for all objects. Finally, to arrive to the objects merits, it makes the normalized sum of the relative importance products.

\section{Multi-Level Solving}

Note that if, for instance, $d(1)$ and $s(1)$ are fixed, then ones obtain a problem which is composed by the entities $O=\{o(i) \mid i=\overline{1,4}\}$, $A=\{a(j) \mid j=\overline{1,3}\}, \quad S A=\{s a(j) \mid j=\overline{1,3}\}$, $W A=\{w a(j) \mid j=\overline{1,3}\}, \quad\left[p e s \_a(j), o p t \_a(j)\right]$, $j=\overline{1,3}$ and $C(d(1), s(1), o(i), a(j))=c_{11 i j}$ where $i=\overline{1,4}, j=\overline{1,3}$ i.e. a classical single expert (decision-maker) and single state of nature assessment problem. This problem represents a part of the array depicted in Figure 1. It is possible to be solved by all chosen methods.

Because of limited space, only the TOPSIS method is going to be detailed below:

Step 1. Normalize the model's data.

To compare the elements of the model, these elements must be normalized, obtaining a new attributes' limits vector and consequences matrix, all together called normalized massives.

According to the MADM mathematical requires, there is necessary to use one of von Neuman - Morgenstern normalization methods, in this case the first one.

Using linear interpolation, one obtains the linear equations system:

$\left\{\begin{array}{l}\alpha \cdot \text { pes }+\beta=0 \\ \alpha \cdot \text { opt }+\beta=1\end{array}\right.$

where $\alpha$ and $\beta$ should be determinated such as the value of normalized data is 0 for pes and the value of normalized data is 1 for opt . Then every element norx of the normalized massives will be given by the formula

nor $x=\frac{x-p e s}{\text { opt }- \text { pes }}$

and therefore, the new pes_a(j),opt_a(j) $j=\overline{1, \boldsymbol{j}}$ and $c_{i j} i=\overline{1, \boldsymbol{i}}, j=\overline{1, \boldsymbol{j}}$ are situated between 0 and 1 .

Step 2. Compute the weighted normalized consequences matrix.

$c_{i j}=c_{i j} \cdot w a_{j}, i=\overline{1, \boldsymbol{i}}, j=\overline{1, \boldsymbol{j}}$

where the product is made between the $j^{\text {th }}$ attribute's value of the $i^{\text {th }}$ object and the $j^{\text {th }}$ attribute weight.

Step 3. Determine the positive and negative ideal solution.

opt ${ }_{j}=\left\{\begin{array}{l}\max _{i}\left(c_{i j}\right) \text { for } s a(j)=\text { 'maximum' } \\ \min _{i}\left(c_{i j}\right) \text { for } s a(j)=\text { 'minimum' }\end{array}\right.$ 
pes $_{j}=\left\{\begin{array}{l}\max _{i}\left(c_{i j}\right) \text { for } s a(j)=\text { 'minimum' } \\ \min _{i}\left(c_{i j}\right) \text { for } s a(j)=\text { 'maximum' }\end{array}\right.$

for all $j=\overline{1, \boldsymbol{j}}$.

Step 4. Compute the separation measure using the Euclidian distance.

$d^{+}{ }_{i}=\sqrt{\sum_{j=1}^{\mathrm{j}}\left(c_{i j}-\text { opt }_{j}\right)^{2}}$
$d^{-}{ }_{i}=\sqrt{\sum_{j=1}^{\mathrm{j}}\left(c_{i j}-\text { pes }_{j}\right)^{2}}$

for all $i=\overline{1, i}$. nature. Also, it is applicable for all set of chosen solving methods, in this case M1, M2, M3, and M4.

Just as that problem was built, if one considers the cartesian product of the sets of experts (decision-makers) and states of nature, then ones can consider all $3 \times 6=18$ two-dimensional problems of size 4-rows and 3-colums. They can be solved using M1, M2, M3, M4, separately but in parallel (see again Figure 1) and therefore the tree is reduced with one level.

Problems' solutions are stored into the array $\left\{C_{i l k}\right\} i=\overline{1,4}, l=\overline{1,6}, k=\overline{1,3}$, in which

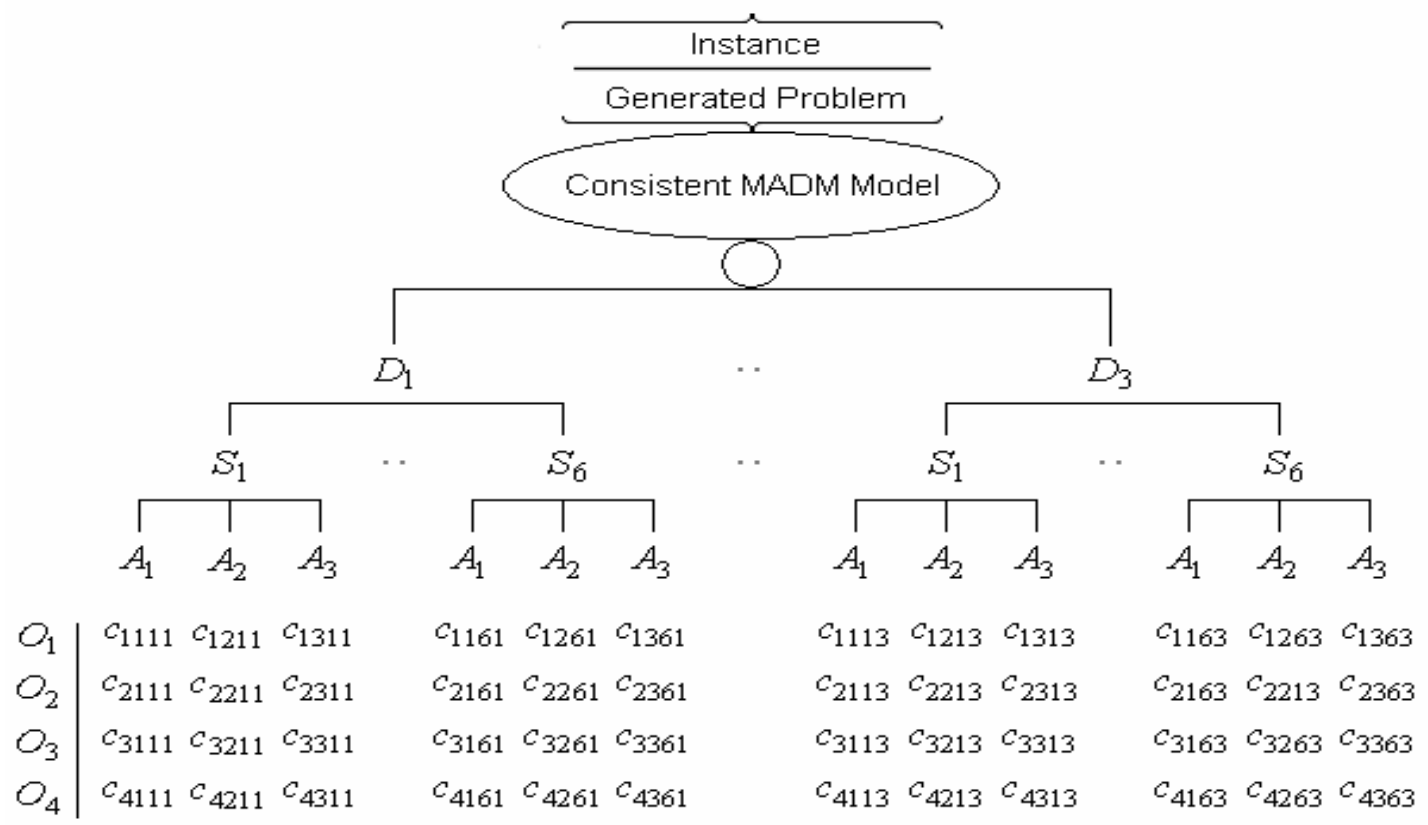

Figure 1. Attributes' level processing

Step 5. Compute the objects merits.

merit $_{i}=\frac{d^{-} i}{d^{+}{ }_{i}-d^{-} i}$

for all $i=\overline{1, i}$.

Step 6. Rank the objects upon their merits. Point the optimum object.

The assessment problems are multi-level problems and in consequence in the following will be provided a procedure for this circumstance. The procedure has as pictorial model a tree, and it is general applicable in the case of multiple experts (decision-makers) and multiple states of the last two subscripts are according to the established hierarchy. This array is transferred to the reduced tree at the states of nature level, which, in this way, becomes the new terminal level.

Similar to the previous level, one also takes into consideration the weights of the states of nature $W S=\{w s(l) \mid l=\overline{1,6}\}$ (see Figure 2). In order to preserve the methodological coherence, as done at the previous step, one continues solving in parallel, using M1, M2, M3, M4, 3 two-dimensional problems of size 4-rows and 6-colums and the tree is reduced again by one level. 


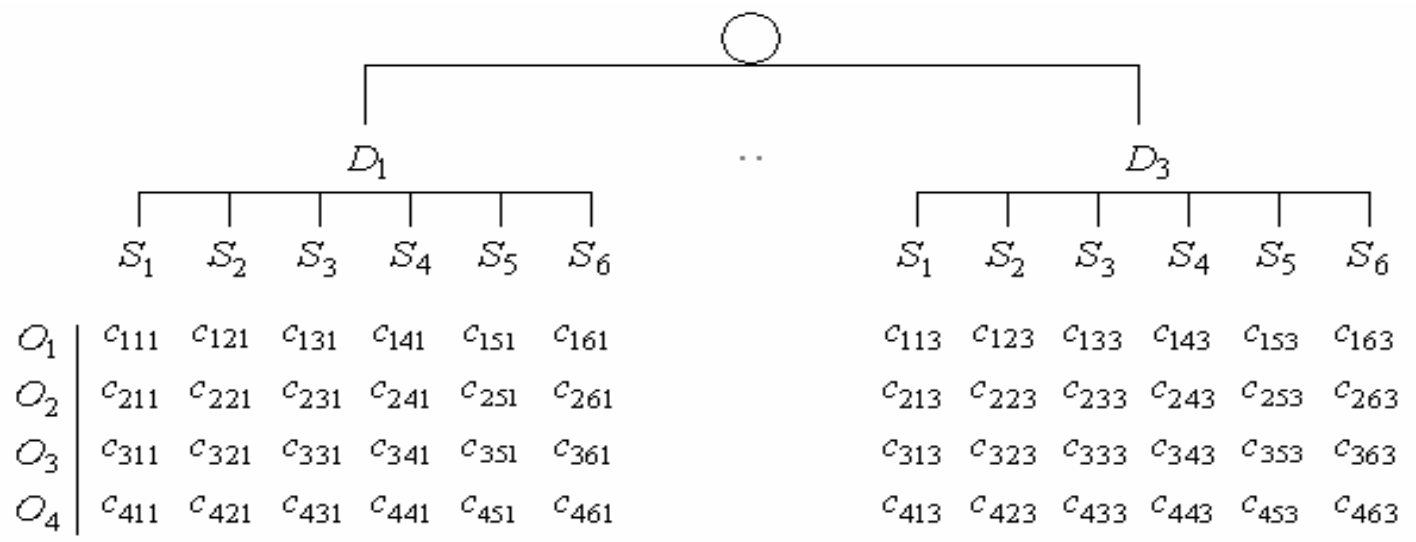

Figure 2. States of nature's level processing

The solutions, which are stored in the array $\left\{C_{i k}\right\} i=\overline{1,4}, k=\overline{1,3}$, are transferred to the reduced tree at the experts (decision-makers) level along with the weights $W D=\{w d(k) \mid$ $k=\overline{1,3}\}$ (see Figure 3).

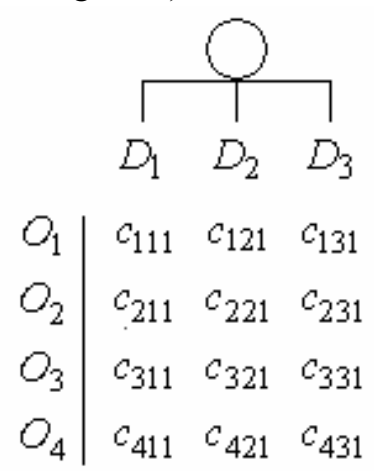

Figure 3. Experts' (decision makers') level processing

In this way, one last two-dimensional problem of size 4-rows and 3-colums needs to be solved, obviously using M1, M2, M3, M4. The solving of this problem produces the final solution $\left\{C_{i}\right\} \quad i=\overline{1,4}$ (see Figure 4, and the corresponding final merit values).

$$
\begin{array}{l|l}
O_{1} & c_{1} \\
O_{2} & c_{2} \\
\sigma_{3} & c_{3} \\
O_{4} & c_{4}
\end{array}
$$

Figure 4. Objects' merits

\section{Assessment Model's Consistency}

In order to verify the consistency of the MADM model, it was considered the first class of generated problems that consists of the problems generated by every physician for every disease stage. A study was made on all resulted merits. If for the same disease stage, the merits, computed starting on the data offered by two physicians, differs by more than 0.2 then a conciliation between the two physicians occurred. In this case, there were some such situations due of wrong surgery costs' appreciation. Obviously, the merits differing by less than 0.2 among the solving methods are considered as a regular situation. For example, let be for analysis two results' matrices given by solving software:

Table 2. Merits matrix for decision-maker $1-$ state of nature I

\begin{tabular}{|l|l|l|l|l|}
\hline merit & M1 & M2 & M3 & M4 \\
\hline$o(1)$ & 0.7500 & 0.5858 & 0.2561 & 0.8686 \\
\hline$o(2)$ & 0.8313 & 0.6936 & 0.2927 & 1.0000 \\
\hline$o(3)$ & 0.7000 & 0.4801 & 0.1951 & 0.0000 \\
\hline$o(4)$ & 0.8500 & 0.5858 & 0.2561 & 0.4114 \\
\hline
\end{tabular}

Table 3. Merits matrix for decision-maker 3 state of nature I

\begin{tabular}{|l|l|l|l|l|}
\hline merit & M1 & M2 & M3 & M4 \\
\hline$o(1)$ & 0.9375 & 0.8649 & 0.3077 & 0.9063 \\
\hline$o(2)$ & 1.0000 & 1.0000 & 0.3297 & 1.0000 \\
\hline$o(3)$ & 0.8500 & 0.5858 & 0.2308 & 0.3750 \\
\hline$o(4)$ & 0.6000 & 0.4142 & 0.1319 & 0.0000 \\
\hline
\end{tabular}

One notes that:

$\mid \operatorname{merit}(M 2, o(1), d(1), s(1)-\operatorname{merit}(M 2, o(1), d(3), s(1) \mid>0.2$

$\mid \operatorname{merit}(M 2, o(2), d(1), s(1)-\operatorname{merit}(M 2, o(2), d(3), s(1) \mid>0.2$

$\mid \operatorname{merit}(M 1, o(4), d(1), s(1)-\operatorname{merit}(M 1, o(4), d(3), s(1) \mid>0.2$

$\mid \operatorname{merit}(M 4, o(3), d(1), s(1)-\operatorname{merit}(M 4, o(3), d(3), s(1) \mid>0.2$

$\mid \operatorname{merit}(M 4, o(4), d(1), s(1)-\operatorname{merit}(M 4, o(4), d(3), s(1) \mid>0.2$ 
These differences are generated by entry data that must be checked-out. Indeed, for example, the consequences matrix for decision-maker 1 and the state of nature 1 , see Table 1, has a wrong element on the position $\mathrm{c} 43$. The cost of surgery in this case is $2200 \mathrm{RON}$ !

Thus the matrix becomes:

Table 4. New consequences matrix for decisionmaker 1 - state of nature I

\begin{tabular}{|l|l|l|l|}
\hline$c$ & $a(1)$ & $a(2)$ & $a(3)$ \\
\hline$o(1)$ & 10 & 10 & 2000 \\
\hline$o(2)$ & 10 & 9 & 1800 \\
\hline$o(3)$ & 10 & 2 & 1800 \\
\hline$o(4)$ & 10 & 2 & 2200 \\
\hline
\end{tabular}

and, in consequence, the new multi-solving provide a new merits matrix:

Table 5. New merits matrix for decision-maker 1 - state of nature I

\begin{tabular}{|l|l|l|l|l|}
\hline merit & M1 & M2 & M3 & M4 \\
\hline$o(1)$ & 0.8750 & 0.7500 & 0.2874 & 0.8814 \\
\hline$o(2)$ & 0.9812 & 0.9301 & 0.3333 & 1.0000 \\
\hline$o(3)$ & 0.8500 & 0.5858 & 0.2414 & 0.4068 \\
\hline$o(4)$ & 0.6000 & 0.4142 & 0.1379 & 0.0000 \\
\hline
\end{tabular}

It is obvious that between this matrix and the merits matrix for decision-maker 3 - state of nature 1 , there are only differences smaller than 0.2 and therefore the model becomes consistent.

Only a consistent MADM model may be at the base of a correct assessment process. As a rule for the multitude of optimizations' approach, first must made the elementary ones and only if the model is consistent then is possible to continue with the others.

\section{Solving Results' Interpretation}

If the results of the first class of MADM problems were analyzed mostly to determine if the model is consistent or not, the results of the last two classes are analyzed by physicians for their medical goals and by the patients to assess the merits of the different surgery schema.

\section{Analysis on stages of the disease}

In order to make an analysis for every disease stage in the opinion of all physicians, it must consider 6 problems, namely all decisionmakers and every state of nature. If the previous 18 basic problems give so called elementary merits matrices, these 6 problems will give onelevel-aggregate merits matrices. In the following all these matrices will be provided with a small comment on objects merits signification, mostly dominance and optimality.

Table 6. Merits matrix for all decision-makers state of nature I

\begin{tabular}{|l|l|l|l|l|}
\hline Merit & M1 & M2 & M3 & M4 \\
\hline$o(1)$ & 0.8500 & 0.7191 & 0.2703 & 1.0000 \\
\hline$o(2)$ & 0.8481 & 0.7423 & 0.2973 & 0.9170 \\
\hline$o(3)$ & 0.6700 & 0.5279 & 0.2162 & 0.0000 \\
\hline$o(4)$ & 0.6900 & 0.5044 & 0.2162 & 0.0437 \\
\hline
\end{tabular}

With two small inadvertences, on M2 and M3 columns, $o(1)$ scheme is the best because it has the best merit upon two solving methods. The $o(2)$ scheme is near to $o(1)$.

Table 7. Merits matrix for all decision-makers state of nature IIA

\begin{tabular}{|l|l|l|l|l|}
\hline merit & M1 & M2 & M3 & M4 \\
\hline$o(1)$ & 0.7762 & 0.6243 & 0.2703 & 0.5826 \\
\hline$o(2)$ & 0.8500 & 0.7521 & 0.3243 & 1.0000 \\
\hline$o(3)$ & 0.4150 & 0.4843 & 0.2162 & 0.0000 \\
\hline$o(4)$ & 0.5362 & 0.4224 & 0.1892 & 0.0770 \\
\hline
\end{tabular}

In this case is obvious that $o(2)$ is the optimum scheme.

Table 8. Merits matrix for all decision-makers state of nature IIB

\begin{tabular}{|l|l|l|l|l|}
\hline merit & M1 & M2 & M3 & M4 \\
\hline$o(1)$ & 0.8125 & 0.6370 & 0.2632 & 0.6141 \\
\hline$o(2)$ & 0.8225 & 0.7972 & 0.3158 & 1.0000 \\
\hline$o(3)$ & 0.2875 & 0.4432 & 0.1842 & 0.0000 \\
\hline$o(4)$ & 0.5860 & 0.5253 & 0.2368 & 0.2893 \\
\hline
\end{tabular}

The expected $o(2)$ scheme is the optimum.

Table 9. Merits matrix for all decision-makers state of nature IIIA

\begin{tabular}{|l|l|l|l|l|}
\hline merit & $\mathrm{M} 1$ & $\mathrm{M} 2$ & $\mathrm{M} 3$ & $\mathrm{M} 4$ \\
\hline$o(1)$ & 0.7160 & 0.5563 & 0.2703 & 0.4835 \\
\hline$o(2)$ & 0.5800 & 0.6891 & 0.3243 & 1.0000 \\
\hline$o(3)$ & 0.3330 & 0.4797 & 0.2162 & 0.3585 \\
\hline$o(4)$ & 0.5514 & 0.4253 & 0.1892 & 0.0000 \\
\hline
\end{tabular}


With one inadvertence, on M1 column, $o(2)$ scheme is optimum. It is true that $o(1)$ dominates on M1 column but it is fort dominated on the rest of columns.

Table 10. Merits matrix for all decision-makers state of nature IIIB

\begin{tabular}{|l|l|l|l|l|}
\hline merit & $\mathrm{M} 1$ & $\mathrm{M} 2$ & $\mathrm{M} 3$ & $\mathrm{M} 4$ \\
\hline$o(1)$ & 0.5600 & 0.5393 & 0.2500 & 0.8477 \\
\hline$o(2)$ & 0.3750 & 0.5503 & 0.2500 & 1.0000 \\
\hline$o(3)$ & 0.6220 & 0.6075 & 0.2778 & 0.9827 \\
\hline$o(4)$ & 0.6175 & 0.4922 & 0.2222 & 0.0000 \\
\hline
\end{tabular}

The expected $o(3)$ scheme is optimum in this case.

Table 11. Merits matrix for all decision-makers state of nature IV

\begin{tabular}{|l|l|l|l|l|}
\hline merit & M1 & M2 & M3 & M4 \\
\hline$o(1)$ & 0.1871 & 0.3312 & 0.1429 & 0.0000 \\
\hline$o(2)$ & 0.4000 & 0.5858 & 0.3143 & 1.0000 \\
\hline$o(3)$ & 0.6633 & 0.5442 & 0.2286 & 0.2525 \\
\hline$o(4)$ & 0.8000 & 0.5879 & 0.3143 & 0.2761 \\
\hline
\end{tabular}

The most indicate scheme for this situation must be $o(4)$ scheme, indeed this object appears as optimum, disregarding M4 column.

\section{Global analysis}

For a global analysis the problem with all experts (decision-makers) and with all disease stages must be considered. The twolevel-aggregate merits' matrix is used only by specialists, because the interpretation is more difficult. By merits globalization, the meaning of merits' optimum is lost. The results express only the belief level in the surgery schema.

Table 12. merits matrix for all decision-makers all states of nature

\begin{tabular}{|l|l|l|l|l|}
\hline merit & $\mathrm{M} 1$ & $\mathrm{M} 2$ & $\mathrm{M} 3$ & $\mathrm{M} 4$ \\
\hline$O(1)$ & 0.6610 & 0.5633 & 0.2432 & 0.4823 \\
\hline$O(2)$ & 0.6144 & 0.6839 & 0.2973 & 1.0000 \\
\hline$O(3)$ & 0.4911 & 0.5199 & 0.2432 & 0.0527 \\
\hline$O(4)$ & 0.6134 & 0.4841 & 0.2162 & 0.0000 \\
\hline
\end{tabular}

\section{Conclusions}

In this paper was presented a real technology to assess the merits of the breast cancer surgery schema. The framework for developing this technology is quite large. One was considered many specialists, stages of the disease and describing attributes. Therefore only a MADM model has been suitable in this case. Talking about MADM problems solving, it is to notice that it was taken into account a multi-solving which is the unique convenient approach.

The used tool was OPTCHOICE software. It allows the following informatics operations: description and validation of the model, generation and multi-solving of the problems.

The breast surgery model once described in the database of OPTCHOICE software, the end users, in this case the physicians, have analyzed the model consistency. It was a useful step in accommodation with the OPTCHOICE software usage. The complex problems, i.e. one-level-aggregate merits problems and two-level-aggregate merits problems, onces generated, were multisolved. The multi-solving proves to be compulsory because. in some cases, using only one mathematical method can lead to unbelievable results. The results were analyzed, step by step, by the physicians involved in this study and the conclusions were very encouraging.

In the same time, an experiment with a patients set was conducted by the specialist physicians. It was a surprise to see that the patients were capable to appreciate, according to their disease stages, and accept the proposed breast surgery schema based on computed merits.

In conclusion, ones can appreciate that the proposed model is a useful tool both for physicians and patients. 


\section{REFERENCES:}

1. GROBSTEIN, R. H., The Breast Cancer Book - What You Need to Know to Make Informed Decisions, Yale Press Log, 2005.

2. PRIBEANU, C., A Usability Assistant for the Heuristic Evaluation of Interactive Systems. Studies in Informatics and Control, Vol. 18(4), December 2009, pp. 355-361.

3. HWANG, C. L., K. YOON, Multiple Attribute Decision Making: Methods and Applications, A state of art Surrey, Springer Verlag, Berlin, 1981.
4. HWANG, C. L., M. J. LIN, Group Decision Making under Multiple Criteria: Methods and Applications. Lecture Notes in Economics and Mathematical Systems, Springer-Verlag, New York, 1987.

5. RESTEANU, C., MADM Theory and practice, Ed. ICI, 2006 (in Romanian).

6. RESTEANU, C., M. ŞOMODI, M. ANDREICA, E. MITAN, Distributed and Parallel Computing in MADM Domain Using the OPTCHOICE Software, Proceedings. of the 7th WSEAS International Conference on Applied Computer Science (ACS'07), Venice, Italy, November 21-23, 2007, pp. 376-384. 